OPEN ACCESS

Edited by:

David W. Ussery,

University of Arkansas for Medical

Sciences, United States

Reviewed by:

Aharon Oren,

Hebrew University of Jerusalem, Israel

Daisuke Shiomi,

Rikkyo University, Japan

Radhey S. Gupta,

McMaster University, Canada

*Correspondence:

Hiroyuki Yamada

hyamada@jata.or.jp

Specialty section: This article was submitted to

Evolutionary and Genomic

Microbiology,

a section of the journal

Frontiers in Microbiology

Received: 21 May 2020 Accepted: 12 October 2020 Published: 16 November 2020

Citation:

Yamada H, Chikamatsu K, Aono A, Murata K, Miyazaki N, Kayama Y, Bhatt A, Fujiwara N,

Maeda S and Mitarai S (2020) Fundamental Cell Morphologies Examined With Cryo-TEM of the Species in the Novel Five Genera

Robustly Correlate With New

Classification in Family

Mycobacteriaceae.

Front. Microbiol. 11:562395. doi: 10.3389/fmicb.2020.562395

\section{Fundamental Cell Morphologies Examined With Cryo-TEM of the Species in the Novel Five Genera Robustly Correlate With New Classification in Family Mycobacteriaceae}

Hiroyuki Yamada1*, Kinuyo Chikamatsu' ${ }^{1}$, Akio Aono' ${ }^{1}$ Kazuyoshi Murata ${ }^{2}$, Naoyuki Miyazaki ${ }^{2,3}$, Yoko Kayama ${ }^{4}$, Apoorva Bhatt ${ }^{5}$, Nagatoshi Fujiwara ${ }^{6}$, Shinji Maeda ${ }^{7}$ and Satoshi Mitarai ${ }^{1,8}$

\footnotetext{
1 Department of Mycobacterium Reference and Research, The Research Institute of Tuberculosis, Japan Anti-Tuberculosis Association, Tokyo, Japan, ${ }^{2}$ Supportive Center for Brain Research, National Institute for Physiological Science, Okazaki, Japan, ${ }^{3}$ Life Science Center for Survival Dynamics, Tsukuba Advanced Research Alliance (TARA), University of Tsukuba, Tsukuba, Japan, ${ }^{4}$ Terabase Inc., Okazaki, Japan, ${ }^{5}$ School of Biosciences and Institute of Microbiology and Infection, University of Birmingham, Birmingham, United Kingdom, ${ }^{6}$ Department of Food and Nutrition, Faculty of Contemporary Human Life Science, Tezukayama University, Nara, Japan, ' Department of Pharmacy, Faculty of Pharmaceutical Science, Hokkaido University of Science, Sapporo, Japan, ${ }^{8}$ Department of Basic Mycobacteriology, Graduate School of Biomedical Sciences, Nagasaki University, Nagasaki, Japan
}

A recent study proposed the novel classification of the family Mycobacteriaceae based on the genome analysis of core proteins in 150 Mycobacterium species. The results from these analyses supported the existence of five distinct monophyletic groups within the genus Mycobacterium. That is, Mycobacterium has been divided into two novel genera for rapid grower Mycobacteroides and Mycolicibacterium, and into three genera for slow grower Mycolicibacter, Mycolicibacillus, and an emended genus Mycobacterium, which include all the major human pathogens. Here, cryo-TEM examinations of 1,816 cells of 31 species (34 strains) belonging to the five novel genera were performed. The fundamental morphological properties of every single cell, such as cell diameter, cell length, cell perimeter, cell circularity, and aspect ratio were measured and compared between these genera. In 50 comparisons on the five parameters between any two genera, only five comparisons showed "non-significant" differences. That is, there are non-significant differences between slow grower genus Mycolicibacillus and genus Mycobacterium in average cell diameter $(p=0.15)$, between rapid grower genus Mycobacteroides and slow grower genus Mycobacterium in average cell length ( $p>0.24$ ), between genus Mycobacteroides and genus Mycobacterium ( $p>0.68$ ) and between genus Mycolicibacter and genus Mycolicibacillus $(p>0.11)$ in average cell perimeter, and between genus Mycolicibacterium and genus Mycobacterium in circularity $(p>0.73)$. The other 45 comparisons showed significant differences between the genera. Genus Mycobacteroides showed the longest average cell diameter, whereas the genus Mycolicibacter showed the shortest average diameter. 
Genus Mycolicibacterium showed the most extended average cell length, perimeter, and aspect ratio, whereas the genus Mycolicibacillus showed the shortest average cell length, perimeter, and aspect ratio. Genus Mycolicibacillus showed the highest average cell circularity, whereas genus Mycobacterium showed the lowest average cell circularity. These fundamental morphological data strongly support the new classification in the family Mycobacteriaceae, and this classification is rational and effective in the study of the members of the family Mycobacteriaceae. Because both the genus Mycolicibacterium and the genus Mycobacterium contain many species and showed larger significant standard deviations in every parameter, these genera may be divided into novel genera which show common genotype and phenotypes in morphology and pathogenicity.

Keywords: family Mycobacteriaceae, cryo-TEM examination, single cell morphology, cell length, cell diameter, circularity, cell perimeter, aspect ratio

\section{INTRODUCTION}

Mycobacterium is often described as " $0.2-0.6 \mu \mathrm{m}$ by $1.0-$ $10.0 \mu \mathrm{m}$, straight or slightly curved rod-shaped bacteria, sometimes branched, acid-fast." Methods of measuring cell morphology include light microscopic examination of stained bacilli or electron microscopy of transmission or scanning. However, both have disadvantages to measure precise values, such as diameter or length, because the resolution power of general light microscopy is approximately $0.2 \mu \mathrm{m}$, which is as large as one-third of the estimated cell diameter, and because measurements with conventional transmission electron microscopy (TEM) and scanning electron microscopy (SEM) examination resulted in smaller values due to the shrinkage of samples during the preparation process due to dehydration and drying (Yamada et al., 2017).

In recent studies on the cell morphology of mycobacteria, bacteria cells were often examined with live time-lapse fluorescent microscopy and the lengths of some cells were measured with microfluidic culture systems (Aldridge et al., 2012; Joyce et al., 2012; Santi et al., 2013; Singh et al., 2013; Wakamoto et al., 2013). Another study measured cell length using a combination of transmission electron microscopy, fluorescent, and light microscopy (Vijay et al., 2014). The same authors examined Mycobacterium tuberculosis (MTB) clinical isolates stained Ziehl-Neelsen and analyzed cell length diversity, which varied from cell to cell (Vijay et al., 2017). However, data obtained from these studies were less precise because of the reasons mentioned above and they rarely measured cell diameters (Milo et al., 2010). This study conducted whole-mount ice-embedded cryo-TEM examinations of more than 1,800 cells of species belonging to the family Mycobacteriaceae, obtaining precise cell morphology data on the cell length, cell diameter, cell perimeter, cell circularity, and aspect ratio.

In 2018, the family Mycobacteriaceae was classified into five genera: the rapidly growing genera Mycobacteroides and Mycolicibacterium, and slow-growing genera Mycolicibacter, Mycolicibacillus, and Mycobacterium, which contain many highly pathogenic species, based on a comparison of core proteins, the average amino acid identity of the conserved protein families, the molecular signatures in the form of conserved signature indels, and conserved signature proteins (Gupta et al., 2018; Oren and Garrity, 2018a,b). Our previous studies reported on a structome analysis of MTB and Mycolicibacterium smegmatis (MSG, basonym Mycobacterium smegmatis), which revealed that non-pathogenic MSG is entirely distinct from virulent MTB (Yamada et al., 2015, 2018). However, MSG has been often used as an alternative to MTB, but MSG is a distinct species in a genus different from MTB (Yamada et al., 2018). The present study was based on the novel classification and comparison of fundamental cell morphology data obtained from cryo-TEM examination between the five genera. Most comparisons of the cell length, the cell diameter, the cell perimeter, the cell circularity, and the aspect ratio revealed significant differences between the five genera. Therefore, these data suggest and support the idea that the novel classification of the family Mycobacteriaceae is proper and reasonable from the viewpoint of cell morphology and the ultimate phenotype.

\section{MATERIALS AND METHODS}

\section{Bacteria}

Bacterial strains examined in this study were purchased from ATCC, JCM, or DSM, except for the strain CDC1551 (Bhatt et al., 2007; Yamada et al., 2012), as listed in Tables 1-3. These bacteria were cultured with $50 \mathrm{ml}$ Middlebrook 7H9 (Becton Dickinson, Sparks, MD, United States) and supplemented with OADC enrichment (oleic acid, bovine albumin (Fraction V), dextrose, and catalase, Becton Dickinson), and 0.05\% Tween 80 (Sigma-Aldrich), contained in a 125-ml Erlenmeyer flask with a flat bottom (Nalgene, 4112-0125, NY, United States) or with Mycobroth (Kyokuto Pharmaceutical Industrial Co. Ltd., Tokyo, Japan).

\section{Preparation of Bacterial Suspensions for Whole-Mount Ice-Embedded CryoTEM Examination}

The cells were then used in the exponential growth phase. Aliquots $(4 \mathrm{ml})$ of cultured cells were transferred to two $2 \mathrm{ml}$ 
TABLE 1 | List of rapid grower species and strains in genus Mycobacteroides and genus Mycolicibacterium examined with cryo-TEM.

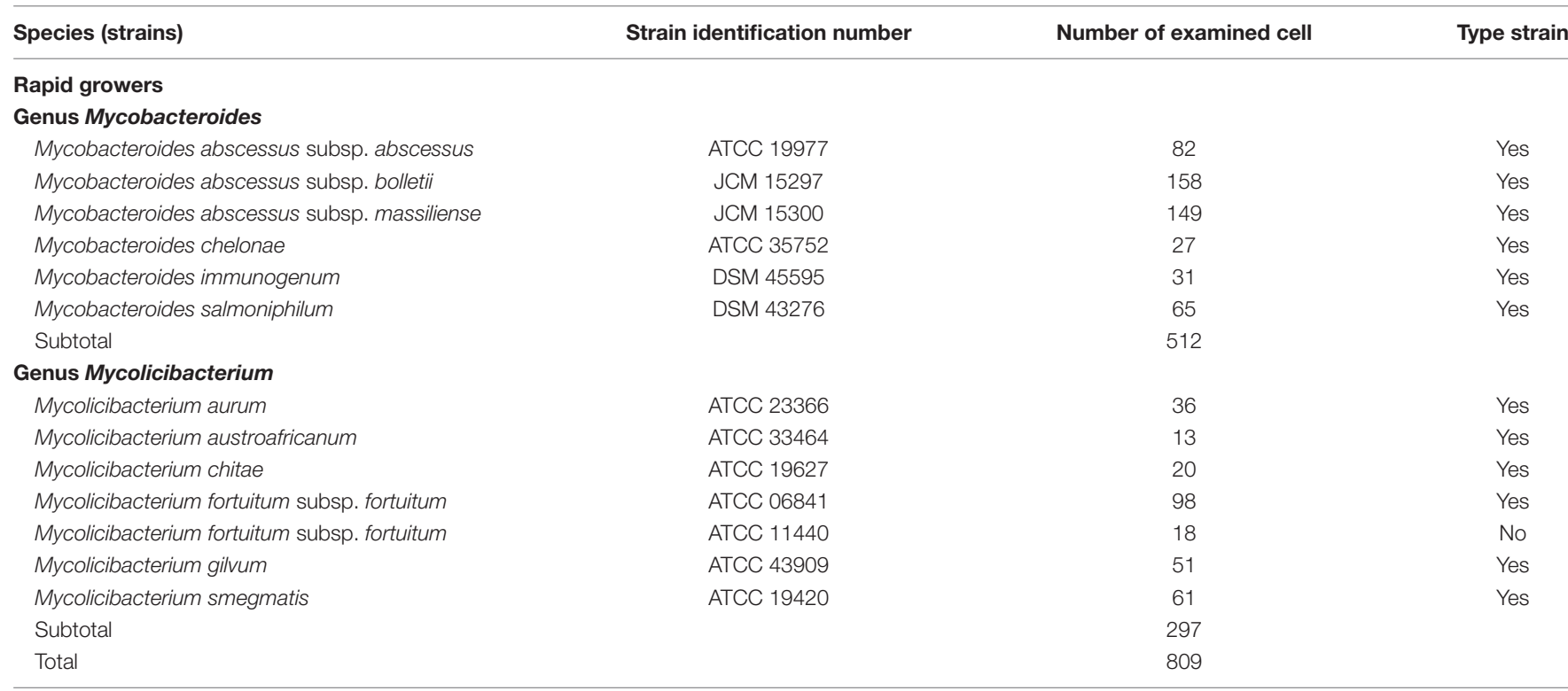

TABLE 2 | List of slow grower species and strains in genus Mycolicibacter and genus Mycolicibacillus examined with cryo-TEM.

\begin{tabular}{|c|c|c|c|}
\hline Species (strains) & Strain identification number & Number of examined cell & Type strain \\
\hline \multicolumn{4}{|l|}{ Slow growers } \\
\hline \multicolumn{4}{|l|}{ Genus Mycolicibacter } \\
\hline Mycolicibacter non chromogenicus & ATCC19530 & 42 & Yes \\
\hline Mycolicibacter terrae & ATCC 15755 & 45 & Yes \\
\hline Subtotal & & 125 & \\
\hline Mycolicibacillus parakoreensis & DSM 45575 & 11 & Yes \\
\hline Mycolicibacillus trivialis & ATCC 23292 & 27 & Yes \\
\hline Subtotal & & 68 & \\
\hline Total & & 193 & \\
\hline
\end{tabular}

TABLE 3 | List of slow grower species and strains in genus Mycobacterium examined with cryo-TEM.

\begin{tabular}{|c|c|c|c|}
\hline Species (strains) & Strain identification number & Number of examined cell & Type stra \\
\hline \multicolumn{4}{|l|}{ Slow growers } \\
\hline \multicolumn{4}{|l|}{ Genus Mycobacterium } \\
\hline Mycobacterium tuberculosis H37Rv & ATCC 27294 & 14 & Yes \\
\hline Mycobacterium tuberculosis CDC1551 & Bhatt et al., 2007 & 29 & No \\
\hline Mycobacterium africanum & ATCC 25420 & 63 & Yes \\
\hline Mycobacterium bovis & ATCC 19210 & 134 & Yes \\
\hline Mycobacterium microti & ATCC 19422 & 28 & Yes \\
\hline Mycobacterium avium & ATCC 25291 & 62 & Yes \\
\hline Mycobacterium celatum & ATCC 51130 & 37 & No \\
\hline Mycobacterium celatum & ATCC 51131 & 25 & Yes \\
\hline Mycobacterium gordonae & ATCC 14470 & 43 & Yes \\
\hline Mycobacterium intermedium & ATCC 51848 & 43 & Yes \\
\hline Mycobacterium intracellulare & ATCC 13950 & 125 & Yes \\
\hline Mycobacterium marinum & ATCC 00927 & 38 & Yes \\
\hline Mycobacterium scrofulaceum & ATCC 19981 & 60 & Yes \\
\hline Mycobacterium ulcerans & ATCC 19423 & 11 & Yes \\
\hline Mycobacterium xenopi & ATCC 19250 & 102 & Yes \\
\hline Total & & 814 & \\
\hline
\end{tabular}


sterile microcentrifuge tubes, which were treated with SigmaCoat (Sigma-Aldrich, SL-2, MO, United States) to prevent bacterial cells from adhering to the tube wall, and centrifuged at $10,000 \times \mathrm{g}$ for $1 \mathrm{~min}$. The supernatants were discarded, and the sediments were resuspended in $2 \mathrm{ml}$ of $2.5 \%$ glutaraldehyde in phosphate buffer (PB, 0.1M, pH7.4) and collected in one microcentrifuge tube. Then the bacterial cells were fixed at $4^{\circ} \mathrm{C}$ overnight. Then, the bacterial suspensions were centrifuged at 10,000 $\times \mathrm{g}$, and the supernatants were discarded and rinsed with $\mathrm{PB}$. After centrifugation at $10,000 \times \mathrm{g}$ and the supernatants were discarded. Finally, the sediments were resuspended with $200 \mu \mathrm{l} \mathrm{PB}$, and the concentrated bacterial suspensions were filtered with Acrodisk $32 \mathrm{~mm}$ Syringe Filter with $5.0 \mu \mathrm{m}$ Supor Membrane (PALL Life Sciences, REF4650, United Kingdom) to obtain singlecell suspensions. The final bacterial solutions were stored at $4^{\circ} \mathrm{C}$ until use. Manipulattions of the bacterial samples before fixation with glutaraldehyde were performed in the biosafety level 3 facility.

\section{Whole-Mount Ice-Embedded CryoTEM Examination}

$1 \mu \mathrm{L}$ of the bacterial suspension prepared above was applied to a glow-discharged carbon grid with holes (Quantifoil copper grids R 2/1 or S 7/2, Quantifoil MicroTools, Jena, Germany) and mounted in an environmentally controlled chamber at $100 \%$ humidity, excess water was removed by blotting, and the grids were frozen in vitreous ice by plunging them into liquid ethane or ethane-propane mixture cooled with liquid nitrogen using a Vitrobot Mark II (FEI, Hillsboro, OR, United States) or EM GP2 (Leica Mikrosysteme GmbH, Vienna, Austria). The grid was loaded in a Single Tilt Liquid Nitrogen Cryo Transfer holder (Model 626, Gatan Inc., Pleasanton, CA, United States) and transferred into TEM.

The microscope was operated at $300 \mathrm{kV}$ (JEM-3100, JEOL, Tokyo, Japan), $200 \mathrm{kV}$ (JEM-2200FS and JEM-2100Plus, JEOL, Tokyo, Japan) or $120 \mathrm{kV}$ (JEM-1230 and JEM-2100Plus, JEOL, Tokyo, Japan) acceleration voltages, and the samples on the grids were cooled during examination with liquid nitrogen in the single tilt nitrogen cryotransfer holder described above at -175.9 to $-177.8^{\circ} \mathrm{C}$. Raw images of the intact cells were recorded at the magnification of $\times 6,000, \times 8,000$, or $\times 10,000$ according to the length of a single cell on a $4 \mathrm{~K} \times 4 \mathrm{~K}$ charge-coupling device (CCD) sensor (F415, TVPIS, Germany) with JEM-3100 and JEM$2200 \mathrm{FS}$, on $1 \mathrm{~K} \times 1 \mathrm{~K}$ CCD digital camera system (OSIS MegaView G2, Olympus, Tokyo, Japan) with JEM-1230, or $2 \mathrm{~K} \times 2 \mathrm{~K}$ high sensitivity CMOS camera system (JEOL, Tokyo, Japan) with JEM-2100Plus. Damaged or bent cells and the cells embedded in the thicker ice were not examined because the exact cell morphology could not be obtained (Yamada et al., 2012, 2018).

\section{Image Analysis}

The captured images were saved as TIFF files, except for the JEM3100 examination, with which images were captured as $\mathrm{dm} 3$ files and converted into TIFF files using Image (Schindelin et al., 2012) plug-in. The cell profiles, which can be traced whole cell outline clearly, were analyzed using the Measure command in the
Analyze menu of ImageJ/Fiji. Briefly, the cell diameter and cell length of each cell were measured using the line selection menu, and cell perimeter, circularity, and the aspect ratio of each cell was measured by tracing the outermost profile of each cell using the polygonal selection menu in the ImageJ window. Measured pixel values were converted to $\mu \mathrm{m}$ or $\mathrm{nm}$ according to the measured pixel value of the scale bar on the corresponding images. In cell length analysis, the shortest and the longest values were recorded, and the longest/the shortest ratios were calculated in each species (each strain). All measures were recorded in Fiji, exported as CSV files, and analyzed with Microsoft ${ }^{\circledR}$ Excel for Mac Ver. 13.36.

\section{Statistics}

Compare mean $t$-test, and one-way ANOVA was performed to compare the differences in mean values. Statistical analyses were performed using StatPlus: mac, AnalystSoft Inc.--statistical analysis program for macOS $^{\circledR}$. Version v7 (StatPlus Mac, RRID:SCR_014635).

\section{RESULTS}

\section{Cryo-TEM Images}

Rapid and slow grower species belonging to 5 genera in the family Mycobacteriaceae were examined cryo-TEM. 1,816 cells of 31 species (34 strains) were examined (Supplementary Figures S4-S8). Representative cryo-TEM images of rapid grower species belonging to genus Mycobacteroides (6 species) and genus Mycolicibacterium (6 species, 7 strains) were shown in Figures 1, 2, respectively. In addition, representative cryoTEM images of slow grower species belonging to genus Mycolicibacter (3 species), genus Mycolicibacillus (3 species), and genus Mycobacterium (13 species, 15 strains) were shown in Figures 3, 4, respectively.

\section{Measurements of Cell Morphological Parameters and Comparison Between the Genera}

Cell diameter, cell length, cell perimeter, circularity, and the aspect ratio of each cell were measured and analyzed with ImageJ/Fiji (Table 4, Figure 5A, and Supplementary Tables S1-S4). In the comparison of the cell diameter, the average value of the whole cells was $0.63 \pm 0.14 \mu \mathrm{m}$. It was extremely similar, where the averages of the genus ranged from $0.49 \pm 0.07$ to $0.69 \pm 0.13 \mu \mathrm{m}$ in genus Mycolicibacter and genus Mycobacteroides, respectively. There are significant differences between five genera $(p<0.000009)$ except for the comparison of the values between slow grower genus Mycolicibacillus and genus Mycobacterium, $0.57 \pm 0.14$ and $0.61 \pm 0.14 \mu \mathrm{m}$, respectively $(p=0.15$, Figure 5B). Because standard deviations (SD) within every genus were small, small $p$-values were calculated despite small differences between genera (Supplementary Table S1).

In the comparison of the cell length, the average value of the whole cells was $2.18 \pm 1.01 \mu \mathrm{m}$, and the average values of each genus varied from genus to genus, where the values ranged from $1.24 \pm 0.47$ to $2.53 \pm 1.48 \mu \mathrm{m}$ in genus Mycolicibacillus and genus 


\section{Genus Mycobacteroides}

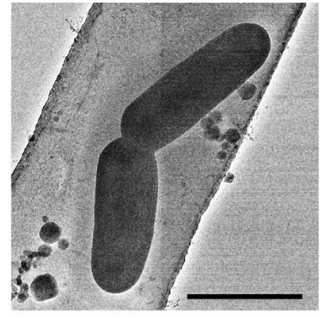

M. abscessus subsp. abscessus

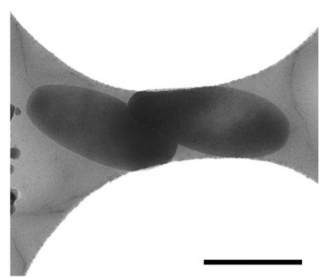

M. chelonae

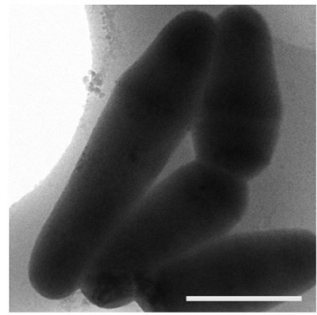

M. abscessus subsp. bolletti

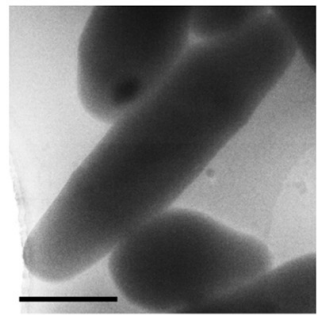

M. immunogenum

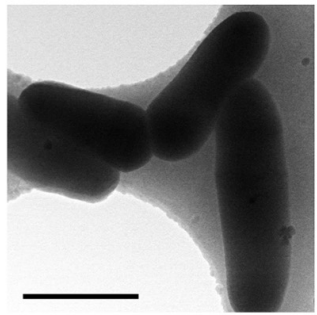

M. abscessus subsp. massiliense

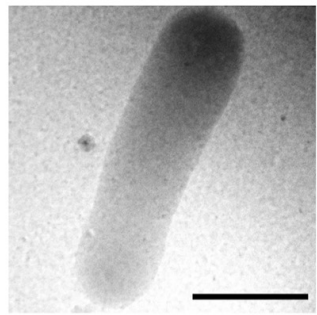

M. salmoniphilum

FIGURE 1 | Representative cryo-TEM images of the cells of rapidly growing species belonging to genus Mycobacteroides; $M$. abscessus subsp. abscessus, M. abscessus subsp. bolletti, M. abscessus subsp. massiliense, M. chelonae, M. immunogenum, and M. salmoniphilum. Each scale bar indicates $1 \mu \mathrm{m}$.

\section{Genus Mycolicibacterium}

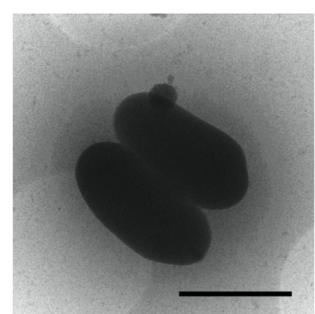

M. aurum

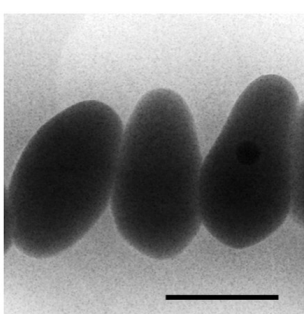

M. austroafricanum

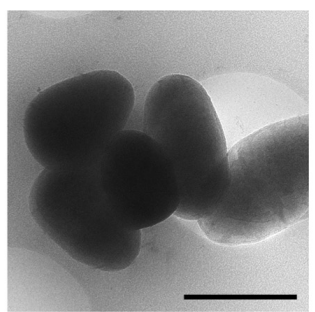

M. chitae

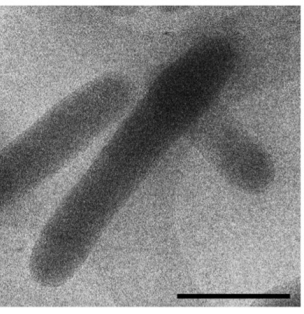

M. fortuitum (ATCC 6841)

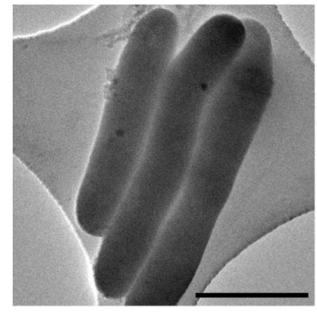

M. fortuitum (ATCC 11440)

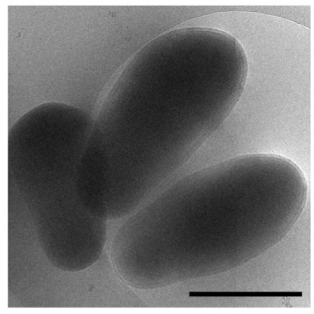

M. gilvum

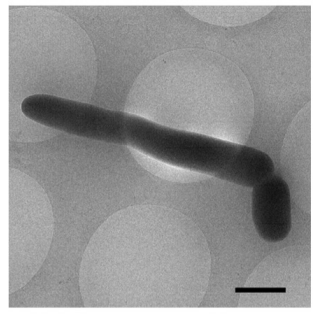

M. smegmatis

FIGURE 2 | Representative cryo-TEM images of the cells of rapidly growing species belonging to genus Mycolicibacterium; M. aurum, M. austroafricanum, M. chitae, M. fortuitum (ATCC 6841), M. fortuitum (ATCC 11440), M. gilvum, and M. smegmatis. Each scale bar indicates $1 \mu m$. 


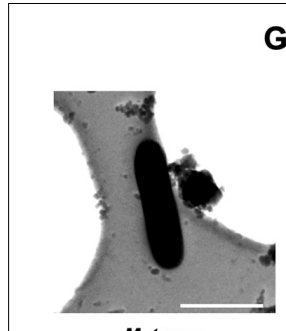

M. terrae

\section{Genus Mycolicibacter}
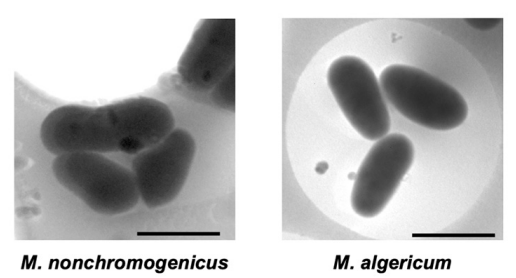

M. algericum

\section{Genus Mycolicibacillus}
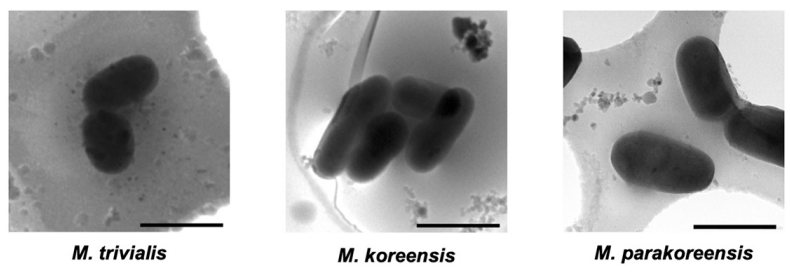

FIGURE 3 | Representative cryoTEM images of the cells of slow-growing species belonging to genus Mycolicibacter, M. terrae, M. non chromogenicus, M. algericum, and genus Mycolicibacillus, M. trivialis, M. koreensis,
M. parakoreensis. Each scale bar indicates $1 \mu \mathrm{m}$.

grower genus Mycobacterium, $5.09 \pm 1.47$ and $5.05 \pm 1.90 \mu \mathrm{m}$ ( $p>0.68)$, respectively, and between slow grower genus Mycolicibacter and genus Mycolicibacillus, $3.38 \pm 1.10$ and $3.12 \pm 0.99 \mu \mathrm{m}(p>0.11)$, respectively (Table 4, Figure 5D, and Supplementary Table S2).

In the comparison of the cell circularity, which was calculated in each cell with the formula, $4 \pi \times$ area/perimeter ${ }^{2}$, measured with Fiji, the average value of the whole cells was $0.65 \pm 0.16$, and the average values of each genus varied from genus to genus with small differences, where the values ranged from $0.62 \pm 0.16$ to $0.82 \pm 0.11$ in genus Mycobacterium and genus Mycolicibacillus, respectively. There are significant differences between five genera $(p<0.00017)$ except for the comparison of the values between rapid grower genus Mycolicibacterium and slow grower genus Mycobacterium, $0.63 \pm 0.21$ and $0.62 \pm 0.16(p>0.73)$, respectively (Table 4, Figure 5E, and Supplementary Table S3).

In the comparison of the cell aspect ratio, the average value of the whole cells was $3.36 \pm 1.59$, and the average values of each genus varied from genus to genus, where the values ranged from $2.11 \pm 0.60$ to $3.80 \pm 2.30$ in genus Mycolicibacillus and genus Mycolicibacterium, respectively. There are significant differences between five genera $(p<0.044)$ without any exception (Table 4 , Figure 5F, and Supplementary Table S4).

\section{DISCUSSION}

between five genera $(p<0.03)$ except for the comparison of the values between rapid grower genus Mycobacteroides and slow grower genus Mycobacterium, $2.19 \pm 0.70$ and $2.25 \pm 0.93 \mu \mathrm{m}$ $(p>0.24)$, respectively (Table 4, Figure 5C, and Supplementary Table S1). Cell length and diameter distributions of single-cell were shown in Supplementary Figure S1.

Cryo-TEM examinations also revealed the large deviations in single-cell length both in the species and in the genus. For example, in the analysis of cell length variation in the examined species, the shortest and the longest cell lengths were extracted, and the ratio of the longest versus the shortest (L/S) was calculated. Then, half of the examined species showed $\mathrm{L} / \mathrm{S}$ value of less than 3.0; the others showed more than 3.0 (Supplementary Figure S2). This means that in half of the examined species the cell divisions occurred asymmetrically. Especially, Mycobacteroides immunogenum, Mycolicibacterium fortuitum subsp. fortuitum (ATCC 6841), Mycobacterium microti, Mycobacterium avium, and Mycobacterium celatum (ATCC 51130), which showed L/S more than 6 (Supplementary Figure S2). This suggests that highly asymmetrical cell division may have occurred in these species. Some examples of the asymmetric cell division were shown in the cryo-TEM images of MSG in Figure 2 and M. celatum (ATCC 51130 and ATCC 51131) in Figure 4 and Supplementary Figures S4-S8.

In the comparison of the cell perimeter, the average value of the whole cells was $4.96 \pm 2.04 \mu \mathrm{m}$, and the average values of each genus varied from genus to genus, where the values ranged from $3.12 \pm 0.99$ to $5.58 \pm 2.99 \mu \mathrm{m}$ in genus Mycolicibacillus and genus Mycolicibacterium, respectively. There are significant differences between five genera $(p<0.002)$ except for the comparison of the values between rapid grower genus Mycobacteroides and slow
1,816 cells belonging to 5 genera in family Mycobacteriaceae were examined in ice-embedded whole-mount cryo-TEM, and the fundamental cell properties were obtained through Fiji/ImageJ software. In a comparison between newly proposed genera (Gupta et al., 2018), there were a substantial number of significant differences in cell diameter, cell length, cell perimeter, circularity, and aspect ratio. However, the deviation in cell diameter was small despite significant differences. The total average was $0.63 \mu \mathrm{m}$ with an SD of $0.14 \mu \mathrm{m}$, which is less than $25 \%$ of the average. Furthermore, the average in three genera Mycobacteroides, Mycolicibacterium, and Mycobacterium ranged from 0.61 to $0.69 \mu \mathrm{m}$, and those of two genera Mycolicibacter and Mycolicibacillus ranged from 0.49 to $0.57 \mu \mathrm{m}$. These data suggest that the cell diameters of the species in the family Mycobacteriacea maybe strictly regulated with the conserved genetical manner by the actin homolog MreB (Table 4; Melzer et al., 2018), as the cell diameters of model bacteria such as E. coli and $B$. subtilis are regulated by the cytoskeletal protein MreB (Dion et al., 2019; Kurita et al., 2019; Patel et al., 2020).

In contrast, there are significant differences in the average cell length between five genera (Table 4). However, the average values of the single species in each genus were not even, but heterogeneous. The cell length varied from not only genus to genus on average, but also species to species within the same genus, and also from cell to cell within the same species (Supplementary Figures S1, S2).

There are two groups of species based on the longest cell length versus the shortest cell length $(\mathrm{L} / \mathrm{S})$ ratio, that is, $\mathrm{L} / \mathrm{S}$ ratio $<3$ species (group 1) and L/S ratio $>3$ species (group 2). As shown in Supplementary Figures S1, S2, in genus Mycobacteroides, 


\section{Genus Mycobacterium}

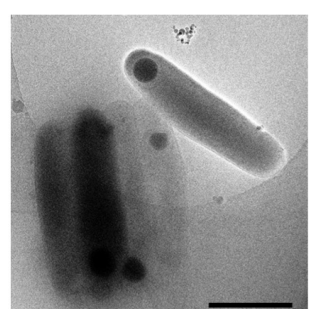

M. tuberculosis (H37Rv)

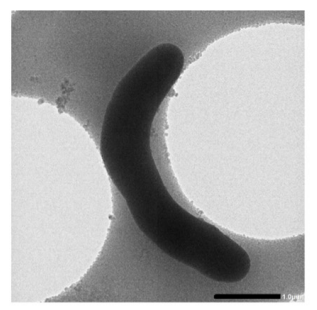

M. microti

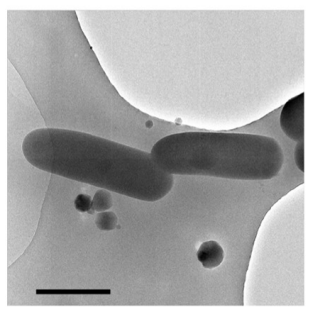

M. gordonae

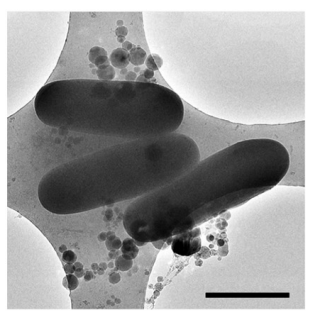

M. scrofulaceum

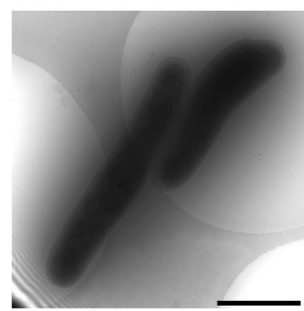

M. tuberculosis (CDC1551)

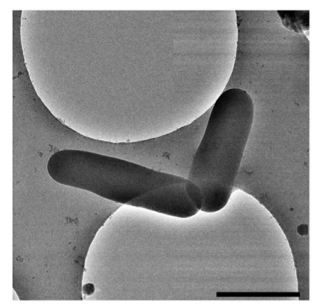

M. avium

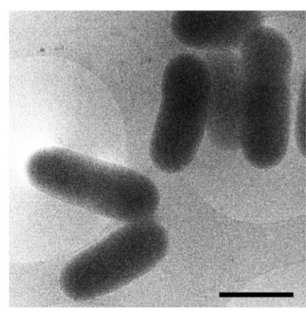

M. intermedium

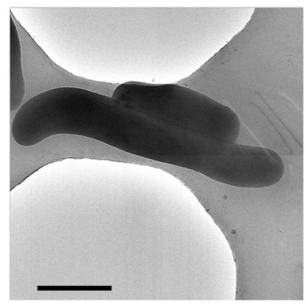

M. ulcerans

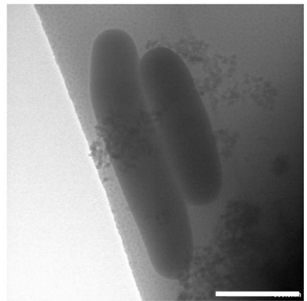

M. africanum

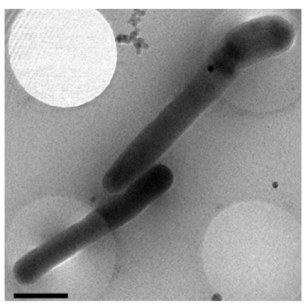

M. celatum

(ATCC 51130)

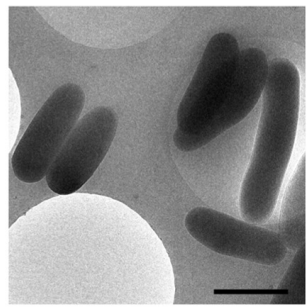

M. intracellulare

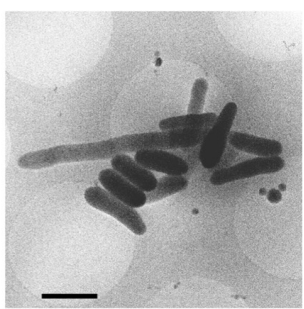

M. xenopi

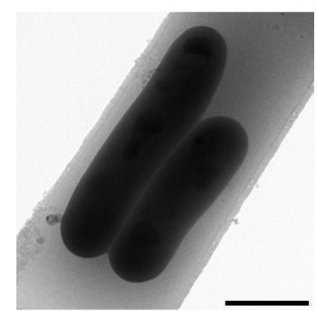

M. bovis

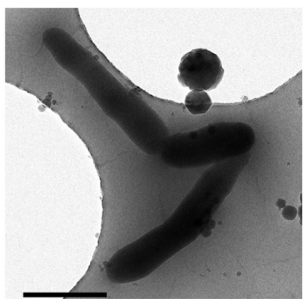

M. celatum

(ATCC 51131)

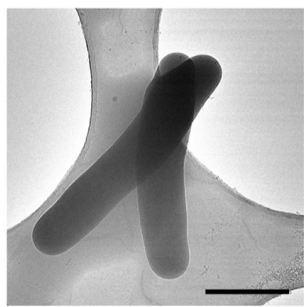

M. marinum

FIGURE 4 | Representative cryo-TEM images of the cells of slow-growing species belonging to genus Mycobacterium, M. tuberculosis (H37Rv), M. tuberculosis (CDC1551), M. africanum, M. bovis, M. microti, M. avium, M. celatum (ATCC 51130), M. celatum (ATCC 51131), M. gordonae, M. intermedium, M. intracellulare, M. marinum, M. scrofulaceum, M. ulcerans, and M. xenopi. Each scale bar indicates $1 \mu \mathrm{m}$.

group 1 vs. group 2 ratio was 3: 3, in genus Mycolicibacterium, that was 4: 3, in genus Mycolicibacter, that was 0: 3, in Mycolicibacillus, that was 2: 1, and in genus Mycobacterium, that was 7: 8. There are some reports about asymmetric cell division in species belonging to genera in the family Mycobacteriaceae (Aldridge et al., 2012; Joyce et al., 2012; Santi et al., 2013; Singh et al., 2013; Wakamoto et al., 2013), such as MSG and MTB. Based on this study, the cells of group 2 species with high
L/S ratio may divide asymmetrically, such as Mycobacteroides immunogenum, Mycolicibacterium fortuitum, species belonging to genus Mycolicibacter, Mycobacterium microti, Mycobacterium avium, Mycobacterium celatum, Mycobacterium intracellulare, Mycobacterium marinum, Mycobacterium scrofulaceum, and Mycobacterium xenopi (Supplementary Figures S1, S2). On the other hand, our data suggest that the cells of group 1 species with a low L/S ratio may divide rather symmetrically. It is known 
TABLE 4 | Comparison of average values in diameter, length, perimeter, circularity, and aspect ratio of the cells between genera.

\begin{tabular}{|c|c|c|c|c|c|}
\hline & \multicolumn{5}{|c|}{ Genus } \\
\hline & \multicolumn{2}{|c|}{ Rapid grower } & \multicolumn{3}{|c|}{ Slow grower } \\
\hline & Mycobacteroides & Mycolicibacterium & Mycolicibacter & Mycolicibacillus & Mycobacterium \\
\hline Species (strains) & 6 & $6(7)$ & 3 & 3 & $13(15)$ \\
\hline Number of examined cells & 512 & 297 & 125 & 68 & 814 \\
\hline Diameter $(\mu \mathrm{m})$ & & & $0.63 \pm 0.14^{\star}$ & & \\
\hline Average & 0.69 & 0.65 & 0.49 & 0.57 & 0.60 \\
\hline SD & 0.13 & 0.11 & 0.07 & 0.14 & 0.13 \\
\hline Minimum & 0.43 & 0.45 & 0.24 & 0.38 & 0.33 \\
\hline Maximum & 1.15 & 1.00 & 0.70 & 0.91 & 1.03 \\
\hline Length ( $\mu \mathrm{m})$ & & & $2.18 \pm 1.01^{\star}$ & & \\
\hline Average & 2.19 & 2.51 & 1.41 & 1.24 & 2.25 \\
\hline SD & 0.70 & 1.46 & 0.55 & 0.47 & 0.93 \\
\hline Shortest & 0.78 & 0.94 & 0.53 & 0.60 & 0.77 \\
\hline Longest & 6.31 & 8.58 & 3.74 & 2.83 & 6.98 \\
\hline L/S ratio & 8.14 & 9.10 & 7.00 & 4.71 & 9.02 \\
\hline Perimeter $(\mu \mathrm{m})$ & & & $4.96 \pm 2.04^{\star}$ & & \\
\hline Average & 5.09 & 5.52 & 3.38 & 3.12 & 5.05 \\
\hline SD & 1.47 & 2.88 & 1.10 & 0.99 & 1.90 \\
\hline Minimum & 2.20 & 2.62 & 1.75 & 1.86 & 2.27 \\
\hline Maximum & 13.8 & 17.8 & 7.99 & 6.49 & 13.6 \\
\hline Circularity & & & $0.65 \pm 0.16^{\star}$ & & \\
\hline Average & 0.67 & 0.63 & 0.71 & 0.82 & 0.62 \\
\hline $\mathrm{SD}$ & 0.11 & 0.21 & 0.13 & 0.11 & 0.16 \\
\hline Minimum & 0.40 & 0.18 & 0.35 & 0.56 & 0.17 \\
\hline Maximum & 0.96 & 0.96 & 0.98 & 0.97 & 0.97 \\
\hline Aspect ratio & & & $3.36 \pm 1.59^{\star}$ & & \\
\hline Average & 3.07 & 3.80 & 2.81 & 2.11 & 3.56 \\
\hline SD & 0.87 & 2.30 & 1.01 & 0.60 & 1.64 \\
\hline Minimum & 1.23 & 1.30 & 0.98 & 1.14 & 1.17 \\
\hline Maximum & 6.22 & 14.4 & 7.21 & 3.79 & 15.8 \\
\hline
\end{tabular}

*Total average and standard deviation.

that some bacteria use a negative regulation of FtsZ assembly, recognized as nucleoid occlusion. However, because it has been shown that the FtsZ of MSG (group 1) can complement the deletion of MTB (group 2), the asymmetric cell divisions are not attributed to the difference of FtsZ in genotype or phenotype between group 1 and group 2 (Hett and Rubin, 2008; Eswara and Ramamurthi, 2017).

It is noteworthy that in genus Mycobacterium, a member of MTB complex, Mycobacterium microti showed significantly longer cell length than MTB (both H37Rv and CDC1551), M. africanum, and $M$. bovis $(p<0.004)$. There were no significant differences in average cell length between MTB H37Rv and CDC1551 $(p>0.6)$, and between MTB (H37Rv and CDC1551) and $M$. africanum $(p>0.3)$. In contrast, there were significant differences between MTB (H37Rv and CDC1551) and $M$. bovis $(p<0.02)$, and between $M$. africanum and $M$. bovis $(p<0.00000003)$ because average cell length in $M$. bovis was the shortest.

Although members of MTB complex have highly conserved genomes, $M$. microti genome lacks RD1 and RD3-RD10, and
MiD1, RD1b, MiD2, and MiD3 (Brodin et al., 2002; Frota et al., 2004; Mostowy et al., 2004). However, genomic deletions of RD1 and RD3-RD10 were also identified in M. bovis BCG. In contrast, $\mathrm{MiD}$ deletions are specific for M. microti, and, MiD3 deletion was universally present to any strains examined (Brodin et al., 2002). Therefore, specific cell morphology in M. microti may be attributed to MiD3 deletion, since MiD3 deletion has been identified in $M$. pinnipedii, the cell morphology of $M$. pinnipedii may validate this conjecture (Supplementary Figure S3).

In the comparison of the cell perimeter and aspect ratio, the differences between the five genera were similar to those of the cell length because the cell perimeters and the aspect ratios were in direct proportion to the cell length. On the other hand, in the comparison of the cell circularity, the differences were inversely correlated with the cell length, where genus Mycolicibacillus, which had the shortest average cell length, showed the highest circularity of the five genera.

In this study, the fundamental cell morphologies of the species belonging to the family Mycobacteriaceae were analyzed based on whole-mount ice-embedded cryo-TEM examinations. There 

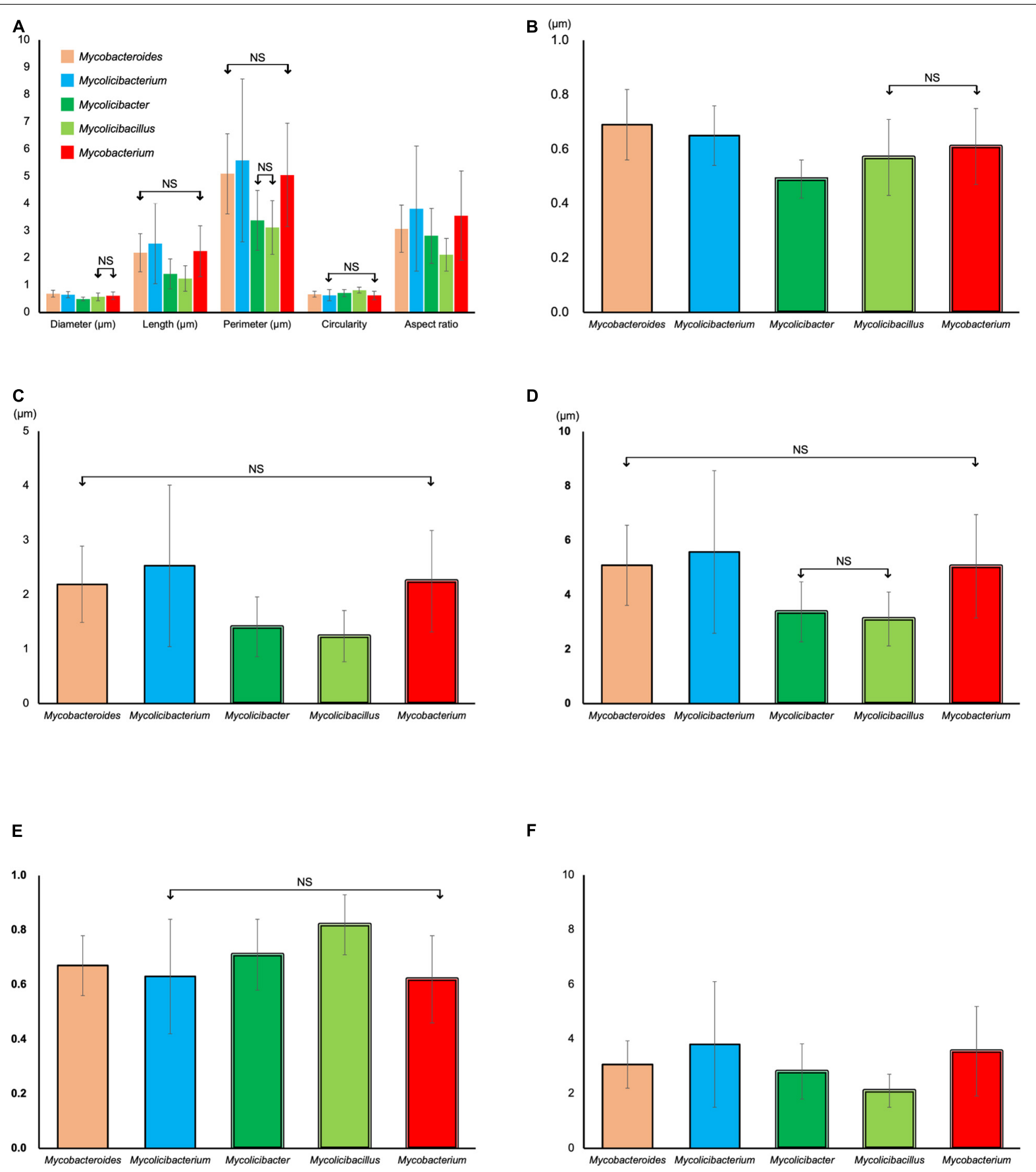

$\mathbf{F}$

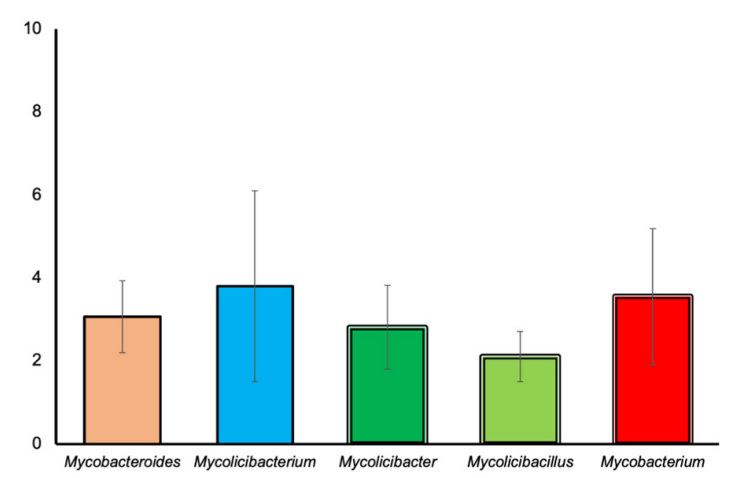

FIGURE 5 | Comparison of the average of the cell diameter, the cell length, the cell perimeter, the cell circularity, and the aspect ratio between genera. (A) Overview of the whole comparison. (B) Comparison of the cell diameter between five genera. (C) Comparison of the cell length between five genera. (D) Comparison of the cell perimeter between five genera. (E) Comparison of the cell circularity between five genera. (F) Comparison of aspect ratio between five genera. Comparisons without significant difference between two genera were indicated as NS. Detail comparison data were shown in Supplementary Tables S1-S4.

have been studies that analyzed cell morphology. However, most of these studies only analyzed cell lengths because it is difficult to precisely analyze cell morphology besides the cell length due to the resolution power of light microscopical examination and to cell shrinkage during sample preparation in the ultrathin section of TEM examination and conventional SEM examination
(Yamada et al., 2015, 2017). Data presented in this study are the first comprehensive quantitative information about the cell morphology belonging to the family Mycobacteriaceae.

Although some studies dispute the novel classification of the family Mycobacteriaceae (Nouioui et al., 2018; Tortoli et al., 2019), others use basonyms (Brzeszcz and Kaszycki, 2018; 
Chevtchouk Jurno et al., 2019), or another study interpreted five novel genera as sub-genera (Bachmann et al., 2019; Matsumoto et al., 2019), the literature supported and used the novel classification (Ito et al., 2018; Yamada et al., 2018; Cereija et al., 2019; Del Barrio-Duque et al., 2019; Kim et al., 2019; Sánchez et al., 2019; Tiago et al., 2019; Zhou et al., 2019; Chin et al., 2020; Salam et al., 2020; Tan et al., 2020). This work reflects comparative morphological studies by Gupta et al. (2018). Although it does not cover all species belonging to four genera Mycobacteroides, Mycolicibacterium, Mycolicibacter, and Mycobacterium, it is one of the most comprehensive morphological analysis to date and includes all the species belonging to the genus Mycolicibacillus. In addition, it is crucial that our data, which showed significant differences in the cell morphology parameters between five genera, strongly support the novel classification of the family Mycobacteriaceae proposed by Gupta et al. (2018) in terms of the cell morphology. Because genus Mycolicibacterium and genus Mycobacterium contain many species with varied morphological properties, these two genera may be further subdivided in future studies.

This study used samples with a single culture period for each species. Because only a small number of cells were examined in some species due to sample preparation immaturity, further investigations are required to provide additional cell morphological data based on the different culture periods and examination with a larger number of cells, especially in two genera Mycolicibacterium and Mycobacterium, both of which contain more than 50 species. Besides, the longer cells may have less chance to be analyzed than the shorter cells because the former may have more chances to localize with overlapping other cells than shorter cells and cannot be accurately traced to the outline. Therefore, the average length, perimeter, and aspect ratio of each species may increase, and the average circularity may decrease according to future investigations. Finally, as we have proposed in our previous report, which undertook a structome analysis in MSG (Yamada et al., 2018), MSG should be interpreted as a completely distinct species from MTB. M. bovis BCG strains or MTB auxotroph strains are more appropriate to use as surrogate species or strains, which can be manipulated in the biosafety level 2 facility (Vilcheze et al., 2018).

\section{REFERENCES}

Aldridge, B. B., Fernandez-Suarez, M., Heller, D., Ambravaneswaran, V., Irimia, D., and Toner, M. (2012). Asymmetry and aging of mycobacterial cells lead to variable growth and antibiotic susceptibility. Science 335, 100-104. doi: 10.1126/ science. 1216166

Bachmann, N. L., Salamzade, R., Manson, A. L., Whittington, R., Sintchenko, V., Earl, A. M., et al. (2019). Key transitions in the evolution of rapid and slow growing mycobacteria identified by comparative genomics. Front. Microbiol. 10:3019. doi: 10.3389/fmicb.2019.03019

Bhatt, A., Fujiwara, N., Bhatt, K., Gurcha, S. S., Kremer, L., Chen, B., et al. (2007). Deletion of kasB in Mycobacterium tuberculosis causes loss of acid-fastness and subclinical latent tuberculosis in immunocompetent mice. Proc. Natl. Acad. Sci. U S A. 104, 5157-5162. doi: 10.1073/pnas.0608654104

Brodin, P., Eiglmeier, K., Marmiesse, M., Billault, A., Garnier, T., Niemann, S., et al. (2002). Bacterial artificial chromosome-based comparative genomic analysis identifies Mycobacterium microti as a natural ESAT-6 deletion mutant. Infect. Immun. 70, 5568-5578. doi: 10.1128/iai.70.10.5568-5578. 2002

\section{DATA AVAILABILITY STATEMENT}

All datasets generated for this study are included in the article/Supplementary Material.

\section{AUTHOR CONTRIBUTIONS}

HY and SMi conceived the project. HY analyzed, interpreted the cryoTEM data, and prepared the figures and manuscript. $\mathrm{SMi}, \mathrm{KC}$, and $\mathrm{AB}$ collected the strains. $\mathrm{HY}, \mathrm{KC}, \mathrm{AA}$, $\mathrm{AB}, \mathrm{NF}$, and SMa cultured bacterial samples. HY, KM, NM, and YK prepared bacterial samples for cryoTEM examination and manage cryoTEM operation and image capturing. All authors had full access to all the data in the study and approved the final version of the manuscript for submission.

\section{FUNDING}

This work has been supported by grants from Research Program on Emerging and Re-emerging Infectious Diseases (JP20fk0108063) and the collaborative programs for National Institute for Physiological Sciences (15-133).

\section{ACKNOWLEDGMENTS}

We are grateful to Prof. Kuniaki Nagayama of the National Institute for Physiological Science for advice at initiating the study.

\section{SUPPLEMENTARY MATERIAL}

The Supplementary Material for this article can be found online at: https://www.frontiersin.org/articles/10.3389/fmicb. 2020.562395/full\#supplementary-material

Brzeszcz, J., and Kaszycki, P. (2018). Aerobic bacteria degrading both n-alkanes and aromatic hydrocarbons: an undervalued strategy for metabolic diversity and flexibility. Biodegradation 29, 359-407. doi: 10.1007/s10532-018-9837-x

Cereija, T. B., Alarico, S., Lourenco, E. C., Manso, J. A., Ventura, M. R., Empadinhas, N., et al. (2019). The structural characterization of a glucosylglycerate hydrolase provides insights into the molecular mechanism of mycobacterial recovery from nitrogen starvation. IUCrJ. 6, 572-585. doi: $10.1107 /$ S2052252519005372

Chevtchouk Jurno, A., Oliveira Correa, Netto, L., Silva Duarte, R., Rocha Pinheiro, and Machado, R. (2019). The search for plant activity against tuberculosis using breakpoints: A review. Tuberculosis 117, 65-78. doi: 10.1016/j.tube.2019.06.004

Chin, K. L., Sarmiento, M. E., Alvarez-Cabrera, N., Norazmi, M. N., and Acosta, A. (2020). Pulmonary non-tuberculous mycobacterial infections: current state and future management. Eur. J. Clin. Microbiol. Infect. Dis. 39, 799-826. doi: 10.1007/s10096-019-03771-0

Del Barrio-Duque, A., Ley, J., Samad, A., Antonielli, L., Sessitsch, A., and Compant, S. (2019). Beneficial endophytic bacteria-Serendipita indica interaction for crop enhancement and resistance to phytopathogens. Front. Microbiol. 10:2888. doi: $10.3389 /$ fmicb. 2019.02888 
Dion, M. F., Kapoor, M., Sun, Y., Wilson, S., Ryan, J., Vigouroux, A., et al. (2019). Bacillus subtilis cell diameter is determined by the opposing actions of two distinct cell wall synthetic systems. Nat. Microbiol. 4, 1294-1305. doi: 10.1038/ s41564-019-0439-0

Eswara, P. J., and Ramamurthi, K. S. (2017). Bacterial cell division: Nonmodels poised to take the spotlight. Ann. Rev. Microbiol. 71, 393-411. doi: 10.1146/ annurev-micro-102215-095657

Frota, C. C., Hunt, D. M., Buxton, R. S., Rickman, L., Hinds, J., Kremer, K., et al. (2004). Genome structure in the vole bacillus, Mycobacterium microti, a member of the Mycobacterium tuberculosis complex with a low virulence for humans. Microbiology 150, 1519-1527. doi: 10.1099/mic.0.26660-0

Gupta, R. S., Lo, B., and Son, J. (2018). Phylogenomics and comparative genomic studies robustly support division of the genus Mycobacterium into an emended genus Mycobacterium and four novel genera. Front. Microbiol. 9:67. doi: 10. 3389/fmicb.2018.00067

Hett, E. C., and Rubin, E. J. (2008). Bacterial growth and cell division: a mycobacterial perspective. Microbiol. Mol. Biol. Rev. 72, 126-156. doi: 10.1128/ MMBR.00028-07

Ito, T., Sawai, K., Kawai, M., Nozaki, K., Otsu, K., Fukushi, H., et al. (2018). Draft genome sequences of Mycolicibacter senuensis isolate GF74 and Mycobacterium colombiense isolates GF28 and GF76 from a swine farm in Japan. Microbiol. Resour. Announc. 7, e918-e936. doi: 10.1128/MRA.00936-18

Joyce, G., Williams, K. J., Robb, M., Noens, E., Tizzano, B., Shahrezaei, V., et al. (2012). Cell division site placement and asymmetric growth in mycobacteria. PLoS One 7:e44582. doi: 10.1371/journal.pone.0044582

Kim, B. J., Kim, G. N., Kim, B. R., Shim, T. S., Kook, Y. H., and Kim, B. J. (2019). New Mycobacteroides abscessus subsp. massiliense strains with recombinant hsp65 gene laterally transferred from Mycobacteroides abscessus subsp. abscessus: Potential for misidentification of $M$. abscessus strains with the hsp65-based method. PLoS One 14:e0220312. doi: 10.1371/journal.pone. 0220312

Kurita, K., Shin, R., Tabei, T., and Shiomi, D. (2019). Relation between rotation of MreB actin and cell width of Escherichia coli. Genes Cells 24, 259-265. doi: 10.1111 /gtc. 12667

Matsumoto, Y., Kinjo, T., Motooka, D., Nabeya, D., Jung, N., Uechi, K., et al. (2019). Comprehensive subspecies identification of 175 nontuberculous mycobacteria species based on 7547 genomic profiles. Emerg. Microbes Infect. 8, 1043-1053. doi: 10.1080/22221751.2019.1637702

Melzer, E. S., Sein, C. E., Chambers, J. J., and Sloan Siegrist, M. (2018). DivIVA concentrates mycobacterial cell envelope assembly for initiation and stabilization of polar growth. Cytoskeleton 75, 498-507. doi: 10.1002/cm.21490

Milo, R., Jorgensen, P., Moran, U., Weber, G., and Springer, M. (2010). BioNumbers-the database of key numbers in molecular and cell biology. Nucleic Acids Res. 38, D750-D753. doi: 10.1093/nar/gkp889

Mostowy, S., Cousins, D., and Behr, M. A. (2004). Genomic interrogation of the dassie bacillus reveals it as anique RD1 mutant within the Mycobacterium tuberculosis complex. J. Bacteriol. 186, 104-109. doi: 10.1128/JB.186.1.104-109. 2004

Nouioui, I., Carro, L., García-López, M., Meier-Kolthoff, J. P., Woyke, T., Kyrpides, N. C., et al. (2018). Genome-based taxonomic classification of the phylum Actinobacteria. Front. Microbiol. 9:2007. doi: 10.3389/fmicb.2018.02007

Oren, A., and Garrity, G. (2018a). List of new names and new combinations previously effectively, but not validly, published. Int. J. Syst. Evol. Microbiol. 68, 1411-1417. doi: 10.1099/ijsem.0.002711

Oren, A., and Garrity, G. (2018b). Notification of changes in taxonomic opinion previously published outside the IJSEM. Int. J. Syst. Evol. Microbiol. 68, 21372138. doi: 10.1099/ijsem.0.002830

Patel, Y., Zhao, H., and Helmann, J. D. (2020). A regulatory pathway that selectively up-regulates elongasome function in the absence of class A PBPs. Elife 9, e57902. doi: $10.7554 /$ eLife.57902

Salam, N., Jiao, J. Y., Zhang, X. T., and Li, W. J. (2020). Update on the classification of higher ranks in the phylum Actinobacteria. Int. J. Syst. Evol. Microbiol. 70, 1331-1355. doi: 10.1099/ijsem.0.003920

Sánchez, M., Blesa, A., Sacristán-Horcajada, E., and Berenguer, J. (2019). Complete genome sequence of Mycolicibacterium hassiacum DSM 44199. Microbiol. Resour. Announc. 8, 1522-1518. doi: 10.1128/MRA.01522-18

Santi, I., Dhar, N., Bousbaine, D., Wakamoto, Y., and McKinney, J. D. (2013). Single-cell dynamics of the chromosome replication and cell division cycles in mycobacteria. Nat. Commun. 4, 2470. doi: 10.1038/ncomms3470
Schindelin, J., Arganda-Carreras, I., Frise, E., Kaynig, V., Longair, M., Pietzsch, T., et al. (2012). Fiji: an open-source platform for biological-image analysis. Nature methods 9, 676-682. doi: 10.1038/nmeth.2019

Singh, B., Nitharwal, R. G., Ramesh, M., Pettersson, B. M., Kirsebom, L. A., and Dasgupta, S. (2013). Asymmetric growth and division in Mycobacterium spp.: compensatory mechanisms for non-medial septa. Mol Microbiol. 88, 64-76. doi: $10.1111 / \mathrm{mmi} .12169$

Tan, W., Liao, T. H., Wang, J., Wei, Y. C., Zhou, H. K., Xiao, Y., et al. (2020). A recently evolved diflavin-containing monomeric nitrate reductase is responsible for highly efficient bacterial nitrate assimilation. J. Biol. Chem. 295, 5051-5066. doi: 10.1074/jbc.RA120.012859

Tiago, I., Alarico, S., Maranha, A., Coelho, C., Goncalves Pereira, S., and Empadinhas, N. (2019). High-quality draft genome sequences of rare nontuberculous mycobacteria isolated from surfaces of a hospital. Microbiol. Resour. Announc. 8, e496-e419. doi: 10.1128/MRA.00496-19

Tortoli, E., Brown-Elliott, B. A., Chalmers, J. D., Cirillo, D. M., Daley, C. L., Emler, S., et al. (2019). Same meat, different gravy: ignore the new names of mycobacteria. Eur. Respir. J. 54, 496-419. doi: 10.1183/13993003.007952019

Vijay, S., Mukkayyan, N., Sebastian, J., and Ajitkumar, P. (2014). Asymmetric cell division in Mycobacterium tuberculosis and its unique features. Arch. Microbiol. 196, 157-168. doi: 10.1007/s00203-014-0953-7

Vijay, S., Vinh, D. N., Hai, H. T., Ha, V. T. N., Dung, V. T. M., Dinh, T. D., et al. (2017). Influence of stress and antibiotic resistance on cell-length distribution in Mycobacterium tuberculosis clinical isolates. Front. Microbiol. 8:2296. doi: 10.3389/fmicb.2017.02296

Vilcheze, C., Copeland, J., Keiser, T. L., Weisbrod, T., Washington, J., Jain, P., et al. (2018). Rational design of biosafety level 2-approved, multidrug-resistant strains of Mycobacterium tuberculosis through nutrient auxotrophy. mBio 9 , e938-e918. doi: 10.1128/mBio.00938-18

Wakamoto, Y., Dhar, N., Chait, R., Schneider, K., Signorino-Gelo, F., Leibler, S., et al. (2013). Dynamic persistence of antibiotic-stressed mycobacteria. Science 4 339, 91-95. doi: 10.1126/science.1229858

Yamada, H., Bhatt, A., Danev, R., Fujiwara, N., Maeda, S., Mitarai, S., et al. (2012). Non-acid-fastness in Mycobacterium tuberculosis $\Delta$ kasB mutant correlates with the cell envelope electron density. Tuberculosis 92, 351-357. doi: 10.1016/j.tube. 2012.02.006

Yamada, H., Yamaguchi, M., Chikamatsu, K., Aono, A., and Mitarai, S. (2015). Structome analysis of virulent Mycobacterium tuberculosis, which survives with only 700 ribosomes per $0.1 \mathrm{fl}$ of cytoplasm. PLoS One 10:e0117109. doi: 10.1371/ journal.pone.011710

Yamada, H., Yamaguchi, M., Igarashi, Y., Chikamatsu, K., Aono, A., Murase, Y., et al. (2018). Mycolicibacterium smegmatis, basonym Mycobacterium smegmatis, expresses morphological phenotypes much more similar to Escherichia coli than Mycobacterium tuberculosis in quantitative structome analysis and cryoTEM examination. Front. Microbiol. 9:1992. doi: 10.3389/fmicb.2018. 01992

Yamada, H., Yamaguchi, M., Shimizu, K., Murayama, S. Y., Mitarai, S., Sasakawa, C., et al. (2017). Structome analysis of Escherichia coli cells by serial ultrathin sectioning reveals the precise cell profiles and the ribosome density. Microscopy 66, 283-294. doi: 10.1093/jmicro/dfx019

Zhou, L., Ma, C., Xiao, T., Li, M., Liu, H., Zhao, X., et al. (2019). A new single gene differential biomarker for Mycobacterium tuberculosis complex and nontuberculosis mycobacteria. Front. Microbiol 10:1887. doi: 10.3389/fmicb.2019. 01887

Conflict of Interest: YK was employed by the company Terabase Inc.

The remaining authors declare that the research was conducted in the absence of any commercial or financial relationships that could be construed as a potential conflict of interest.

Copyright (C) 2020 Yamada, Chikamatsu, Aono, Murata, Miyazaki, Kayama, Bhatt, Fujiwara, Maeda and Mitarai. This is an open-access article distributed under the terms of the Creative Commons Attribution License (CC BY). The use, distribution or reproduction in other forums is permitted, provided the original author(s) and the copyright owner(s) are credited and that the original publication in this journal is cited, in accordance with accepted academic practice. No use, distribution or reproduction is permitted which does not comply with these terms. 